\title{
A TEST OF PRE-MAIN-SEQUENCE LITHIUM DEPLETION MODELS
}

\author{
JeNNIFER C. YeE ${ }^{1}$ AND ERIC L. N. JeNSEN ${ }^{2}$ \\ ${ }^{1}$ Department of Astronomy, Ohio State University, 140 West 18th Avenue, Columbus, OH 43210, USA \\ ${ }^{2}$ Department of Physics and Astronomy, Swarthmore College, 500 College Avenue, Swarthmore, PA 19081, USA \\ Received 2009 August 26; accepted 2010 January 20; published 2010 February 9
}

\begin{abstract}
Despite the extensive study of lithium depletion during pre-main-sequence (PMS) contraction, studies of individual stars show discrepancies between ages determined from the Hertzsprung-Russell (H-R) diagram and ages determined from lithium depletion, indicating open questions in the PMS evolutionary models. To further test these models, we present high-resolution spectra for members of the $\beta$ Pictoris Moving Group (BPMG), which is young and nearby. We measure equivalent widths of the $6707.8 \AA \mathrm{Li}$ I line in these stars and use them to determine lithium abundances. We combine the lithium abundance with the predictions of PMS evolutionary models in order to calculate a lithium depletion age for each star. We compare this age to the age predicted by the $\mathrm{H}-\mathrm{R}$ diagram of the same model. We find that the evolutionary models underpredict the amount of lithium depletion for the BPMG given its nominal H-R diagram age of $\sim 12 \mathrm{Myr}$, particularly for the mid-M stars, which have no observable Li I line. This results in systematically older ages calculated from lithium depletion isochrones than from the H-R diagram. We suggest that this discrepancy may be related to the discrepancy between measured M-dwarf radii and the smaller radii predicted by evolutionary models.
\end{abstract}

Key words: open clusters and associations: individual ( $\beta$ Pictoris Moving Group) - stars: abundances - stars: activity - stars: evolution - stars: late-type - stars: pre-main sequence

Online-only material: color figures

\section{INTRODUCTION}

Lithium depletion during pre-main-sequence (PMS) contraction has been extensively studied. Studies of open clusters have shown that lithium depletion is a strong function of both age and stellar mass (e.g., Soderblom et al. 1993; Barrado y Navascués et al. 2004; Mentuch et al. 2008). These studies clearly show that the presence of a strong Li I line at $6707.8 \AA$ is an indicator of youth in late-type stars. The regularity of lithium depletion in open clusters implies that the lithium line can act as a mass-dependent clock (Jeffries \& Naylor 2001). By combining a measurement of the lithium abundance of a star with theoretical evolutionary models, one might be able to derive an age of a PMS star whose distance is unknown, e.g., a young field star. In order to do this, we need to understand PMS lithium depletion very well.

Most of the work comparing lithium depletion to Hertzsprung-Russell (H-R) diagram ages has been done in open clusters, focusing on comparing the cluster age derived from the lithium depletion boundary (LDB) ${ }^{3}$ in the lowestmass stars with the $\mathrm{H}-\mathrm{R}$ diagram age found from fitting the upper main-sequence (e.g., Barrado y Navascués et al. 1999; Stauffer et al. 1999; Burke et al. 2004; Jeffries \& Oliveira 2005; Manzi et al. 2008). Most studies find that the LDB age tends to be older than the age calculated from upper main-sequence fitting, though the discrepancy may be smaller for younger clusters (Jeffries \& Oliveira 2005; Manzi et al. 2008). These results are used to argue for the inclusion of convective overshooting in evolutionary models, since it increases the ages derived from upper main-sequence fitting without affecting the LDB age. These older upper main-sequence fitting ages tend to be in better agreement with LDB ages (Burke et al. 2004).

\footnotetext{
3 For stars that are fully convective before they reach the main sequence $\left(M<0.3 M_{\odot}\right)$, at a given age there is a sharp boundary between stars that have fully depleted their lithium and slightly less massive stars with no evidence of depletion (Basri et al. 1996; Bildsten et al. 1997).
}

A few studies have been done of individual stars, showing that ages derived from comparison of lithium abundances with models of lithium depletion are persistently older than ages derived from the H-R diagram. Song et al. (2002) find that the age of the binary HIP 112312 derived from its lithium depletion is $>20$ Myr. They find that this system is likely a member of the $\beta$ Pictoris Moving Group (BPMG), whose proposed age derived from PMS isochrones on the $\mathrm{H}-\mathrm{R}$ diagram is $\sim 12 \mathrm{Myr}$ (Zuckerman et al. 2001). Likewise, White \& Hillenbrand (2005) find that the age of St 34 derived from its position on the $\mathrm{H}-\mathrm{R}$ diagram is $8 \pm 3 \mathrm{Myr}$ whereas its lithium depletion implies an age of $>35$ Myr. These results indicate some open questions about lithium depletion in the evolutionary models.

In order to avoid confusing lithium depletion effects with temperature- or mass-dependent systematic trends in the models, we would like to compare H-R diagram ages with lithium depletion ages for individual stars as was done for HIP 112312 and St 34, rather than comparing the ages of more massive stars determined from one technique with the ages of less massive stars determined from another. To do this, we need a coeval group of late-type stars so we can study lithium depletion over a range of masses. We want the stars to be of intermediate age, $\sim 5-80 \mathrm{Myr}$; much younger, and very little lithium depletion has occurred; much older, and they are on the main sequence, where the $\mathrm{H}-\mathrm{R}$ diagram age is degenerate. We also would like them to be nearby, so we can measure accurate distances and luminosities, and bright, so we can get high signal-to-noise ratio spectra.

In this paper, we examine lithium depletion in some of the later-type members of the BPMG, which is ideal for studying lithium depletion because it is young and nearby $(\sim 12 \mathrm{Myr}, 10$ $50 \mathrm{pc}$; Zuckerman et al. 2001). We compare the lithium depletion of our sample to the predictions of different theoretical PMS models. We also calculate ages from these models for individual stars and compare the $\mathrm{H}-\mathrm{R}$ diagram age of each star with the age implied by its lithium depletion to see if each model is internally consistent in the two ages determined for a given star. We 

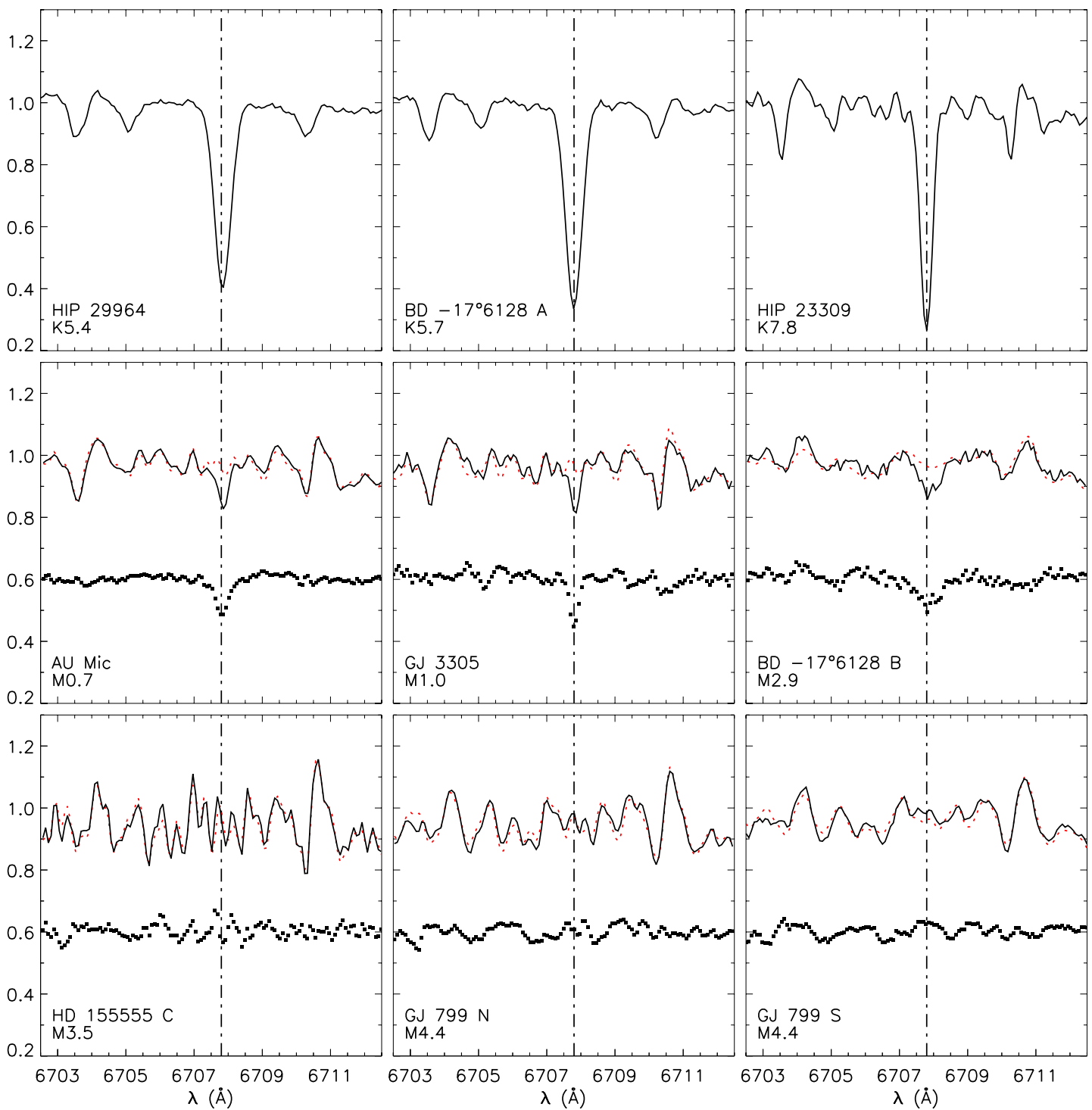

Figure 1. Spectra of BPMG members in the vicinity of the Li line $(\lambda=6707.8 \AA)$; the line's position is marked as a vertical line. The solid curve shows the observed spectrum. For the M stars, the dotted line (red in the online edition) is the spectrum of a main-sequence template of similar spectral type that has been artificially broadened and shifted to produce the best match with the observed spectrum. Filled squares show the difference between the broadened template and the observed spectrum, on the same scale as the observations but with an offset of 0.6 added for clarity. The Li line is clearly detected in the three stars with spectral types earlier than M3 (middle row), but it is not present in the later-type stars (bottom row).

(A color version of this figure is available in the online journal.)

begin by presenting our spectra and lithium equivalent widths (EWs) in Section 2. We then determine effective temperatures, luminosities, and lithium abundances for the stars in our sample in Section 3. In Section 4, we compare these data with the $\mathrm{H}-\mathrm{R}$ diagram and lithium depletion isochrones of theoretical models. We find that the models systematically underpredict the observed lithium depletion for $\mathrm{M}$ stars (or equivalently, overpredict their lithium depletion ages compared to $\mathrm{H}-\mathrm{R}$ diagram ages). We suggest that the observed tendency for models of main-sequence $\mathrm{M}$ stars to underpredict stellar radii may hold the key to resolving the discrepancy between the observed and predicted lithium depletion in late-type stars. We conclude in Section 6.

\section{OBSERVATIONS AND DATA}

We took spectra of ten of the late-K and $\mathrm{M}$ stars or binary systems listed in Table 1 of Zuckerman et al. (2001), with separate spectra for each of the components of the binary stars GJ 799 and $\mathrm{BD}-17^{\circ} 6128$; the spectra are shown in Figures 1 and 2. We also took a spectrum of a somewhat earlier-type member of the BPMG, the spectroscopic binary (SB) HD 155555 A/B. The spectra were taken with the $4 \mathrm{~m}$ Blanco telescope at Cerro Tololo Inter-American Observatory on six consecutive nights between 2001 October 30 and 2001 November 5, using the echelle spectrograph. The spectrograph covered a wavelength range from $4800 \AA$ to $8400 \AA$ with a measured spectral resolving power of $R \sim 40,000$. The spectra were reduced in $\mathrm{IRAF}^{4}$ according to standard procedures. We flux calibrated the spectra with respect to two flux standards, HR 9087 and HR 1544, using standard, sensfunc, and calibrate in IRAF. We visually examined the flux calibrated spectra to

4 IRAF is distributed by the National Optical Astronomy Observatories, which are operated by the Association of Universities for Research in Astronomy, Inc., under cooperative agreement with the National Science Foundation. 


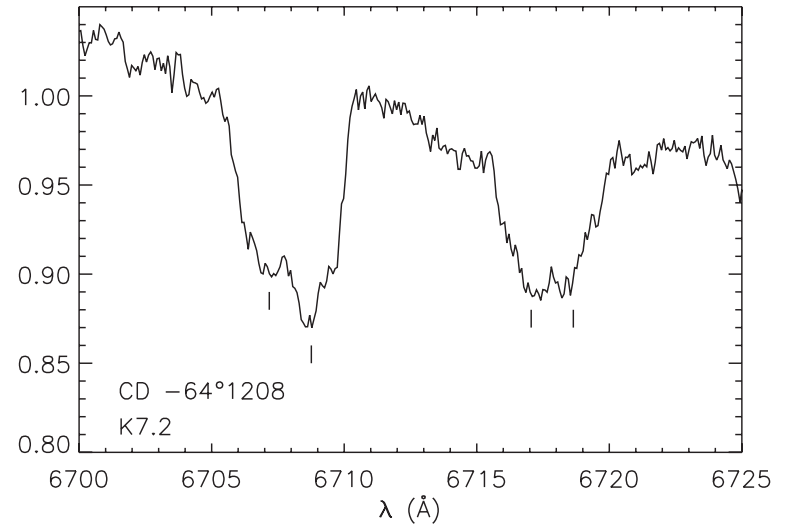

Figure 2. Spectrum of CD $-64^{\circ} 1208$ showing the $\mathrm{Li}$ I $(6707.8 \AA)$ and $\mathrm{Ca}$ I (6717.7 ̊) lines. Note that each line appears to have two components, shown by tick marks at measured RVs of -28 and $43 \mathrm{~km} \mathrm{~s}^{-1}$.

ensure that the calibration was consistent between orders and between the two calibration standards.

\subsection{Spectral Type}

To determine spectral types for our sample, we compared the strength of the TiO5 band to the strength of the nearby continuum, following the method given in Reid et al. (1995). We calculated the ratio of the flux from $7126 \AA$ to $7135 \AA$ to the flux from $7042 \AA$ to $7046 \AA$. Using the linear fit relation from Reid et al. (1995), we took the ratio of these fluxes to determine the spectral types of our sample stars. According to Reid et al. (1995), this method gives an uncertainty of \pm 0.5 spectral subtype. Note that this relation is not well defined for spectral types much earlier than $\mathrm{M} 0$ because the TiO band heads are very weak or disappear entirely. Thus, the spectral types measured for HIP 29964 , BD $-17^{\circ} 6128$ A, and CD $-64^{\circ} 1208$ are more uncertain, although probably correct in a relative sense. Additionally, since the relation gives a numeric value for the spectral type, with 0 representing $\mathrm{M} 0$, it is not clear how to assign that number to the Morgan-Keenan system for values $\leqslant-1$ since some authors argue that K6 is not a distinct spectral type (e.g., Kirkpatrick et al. 1991). We follow the convention of omitting K6 as a spectral type and assign -1 to $\mathrm{K} 7$ and -2 to K5. Thus, we give the spectral types of HIP 29964 and $\mathrm{BD}-17^{\circ} 6128 \mathrm{~A}$ as K5.4 and K5.7, respectively, corresponding to -1.6 and -1.3 as measured from the relation given in Reid et al. (1995). Zuckerman et al. (2001) give the spectral types of these stars as K6/7 and K7/M0, respectively, and Torres et al. (2006) find K4 and K6. The spectral types of the stars later than K7 agree within the errors with the types given in Zuckerman \& Song (2004), with the exception of HD 155555 C, for which Zuckerman \& Song (2004) give M4.5. However, our value of M3.5 for this star is in agreement with the M3 spectral type found by Torres et al. (2006). BD $-17^{\circ} 6128 \mathrm{~B}$ was not listed independently in Zuckerman \& Song (2004), but an estimate of its spectral type is given in Neuhäuser et al. (2002) as M0-2, which is close to the spectral type of M2.9 that we find here.

\subsection{Equivalent Width of Li I}

Using the splot routine in IRAF, we measured the EWs of all identifiable Li I lines at $6707.8 \AA(\mathrm{EW}(\mathrm{Li}$ I $)) .{ }^{5}$ No attempt

\footnotetext{
5 The spectra of M dwarfs are so rich with lines that the observed pseudo-continuum is significantly below the true continuum level. Because of this, for these stars we actually measure a pseudo-EW rather than a true
}

was made to deblend the $\mathrm{Fe}_{\mathrm{I}}$ line at $6707.4 \AA$ from the Li I line. This should not affect our later analyses of $\mathrm{Li}$ abundances since curves of growth for the coolest stars are often derived from measuring the blended lines in model spectra, and thus they also include in the quoted $\mathrm{EW}(\mathrm{Li})$ values the contribution of the Fe I line. The uncertainties given reflect the difference between choosing the height of the continuum to be the maxima or the mean of nearby features. The Li I line is not detected in HD 155555 C, GJ 799 N, or GJ 799 S. Figure 1 shows a comparison of the spectra of the M dwarfs with spectra of mainsequence stars of similar spectral type that have been rotationally broadened to match the observed projected rotational velocity $(v \sin i)$. The difference spectra show that while the line is weak in the early-M stars, it is definitely detected, whereas it is clearly not present in the mid-M dwarfs. We measure upper limits in the $\mathrm{EW}(\mathrm{Li})$ of $30 \mathrm{~m} \AA$ for HD $155555 \mathrm{C}, 10 \mathrm{~m} \AA$ for GJ $799 \mathrm{~N}$, and $10 \mathrm{~m} \AA$ for GJ $799 \mathrm{~S}$.

\section{ANALYSIS}

\subsection{Luminosity and Rotational Velocity}

We determined the luminosity and projected rotational velocity $(v \sin i)$ of each star based on data from the literature. We calculated the luminosity of each star using $V$ magnitudes from Table 1 in Zuckerman et al. (2001). We assigned a fixed value of $\sigma_{V}= \pm 0.1$ as the error on the $V$ magnitudes to account for measurement errors and variability. We used bolometric corrections from Kenyon \& Hartmann (1995) appropriate to the spectral type of the star. Distances to each star came from the new reduction of the Hipparcos catalog (van Leeuwen 2007). The $V$ magnitude of $\mathrm{BD}-17^{\circ} 6128$ is the magnitude of the combined system, so we adopt the luminosities given in Neuhäuser et al. (2002) for the A and B components.

For the $v \sin i$ of each star, we used the average of the values given in Zuckerman et al. (2001), Torres et al. (2006), and Scholz et al. (2007). For CD $-64^{\circ} 1208$, we used the value given in Torres et al. (2006), $v \sin i=110 \mathrm{~km} \mathrm{~s}^{-1}$, as Zuckerman et al. (2001) simply give $v \sin i \gtrsim 100 \mathrm{~km} \mathrm{~s}^{-1}$. BD $-17^{\circ} 6128$ $\mathrm{B}$ does not have a $v \sin i$ given in the literature. We measured the $v \sin i$ by artificially broadening a template star spectrum of similar spectral type (Figure 1). We find that the $v \sin i$ of $\mathrm{BD}-17^{\circ} 6128 \mathrm{~B}$ is $20 \pm 5 \mathrm{~km} \mathrm{~s}^{-1}$.

\subsection{Effective Temperature}

We converted the measured spectral type for each star into an effective temperature $T_{\text {eff }}$ using empirical spectral type- $-T_{\text {eff }}$ relations to calculate the effective temperature of each star. For spectral types M1-M9, we used the temperature scale given in Luhman (1999) for "intermediate" stars, since our young stars can be expected to fall in this class. We combined this with the table given in Kenyon \& Hartmann (1995) for earlier spectral types and interpolated to get effective temperatures for our stars. Changes within the spectral type uncertainties lead to typical changes in effective temperature of $\pm \sim 100 \mathrm{~K}$. These data are given in Table 1.

\subsection{Lithium Abundances}

We calculated lithium abundances $(A(\mathrm{Li})=12+$ $\log (N(\mathrm{Li}) / N(\mathrm{H}))$; Jeffries 2000) for our stars using the curves

equivalent width. For simplicity, we use the term "equivalent width" to refer to both equivalent widths (as measured for $\mathrm{K}$ and earlier spectral types) and pseudo-EW (as measured for the M dwarfs). 
Table 1

Late-type Members of the BPMG

\begin{tabular}{|c|c|c|c|c|c|c|c|c|c|c|}
\hline Name & Other Name & $\begin{array}{c}\text { Spectral } \\
\text { Type }\end{array}$ & $\begin{array}{c}\text { Distance } \\
(\mathrm{pc})\end{array}$ & $\begin{array}{c}V \\
(\mathrm{mag})\end{array}$ & $\begin{array}{c}v \sin i \\
\left(\mathrm{~km} \mathrm{~s}^{-1}\right)\end{array}$ & $\begin{array}{l}T_{\text {eff }} \\
(K)\end{array}$ & $\begin{array}{c}L_{\star} \\
\left(L_{\odot}\right)\end{array}$ & $\begin{array}{c}\mathrm{EW}(\mathrm{Li} \mathrm{I}) \\
(\mathrm{m} \AA)\end{array}$ & $\mathrm{A}(\mathrm{Li})$ & Notes \\
\hline HD $155555 \mathrm{~A}$ & V824 Ara & G5 IV & $31.4 \pm 0.5$ & 7.21 & 34 & $5770_{-110}^{+50}$ & $1.1 \pm 0.1$ & $120 \pm 20$ & $\cdots$ & $\overline{1,2,4}$ \\
\hline HD 155555 B & V824 Ara & K0 IV/V & $31.4 \pm 0.5$ & 8.08 & 33 & $5250_{-90}^{+125}$ & $0.54 \pm 0.06$ & $250 \pm 60$ & $\cdots$ & $1,2,4$ \\
\hline HIP 29964 & AO Men & K5.4 & $38.6 \pm 1.3$ & 9.77 & 15 & $4250_{-150}^{+140}$ & $0.27 \pm 0.04$ & $370 \pm 40$ & $2.5 \pm 0.3$ & $1,2,3$ \\
\hline $\mathrm{BD}-17^{\circ} 6128 \mathrm{~A}$ & HD 358623 A & K5.7 & $45.7 \pm 1.6^{\mathrm{a}}$ & $10.6^{\mathrm{b}}$ & 14 & $4130_{-130}^{+150}$ & $0.25 \pm 0.025^{\mathrm{c}}$ & $410 \pm 20$ & $2.6 \pm 0.2$ & $1,2,5$ \\
\hline $\mathrm{CD}-64^{\circ} 1208$ & $\cdots$ & K7.2 & $28.6 \pm 0.2$ & 9.54 & 110 & $4020_{-110}^{+140}$ & $0.21 \pm 0.04$ & $460 \pm 40$ & $2.7 \pm 0.2$ & 1,2 \\
\hline HIP 23309 & $\mathrm{CD}-57^{\circ} 1054$ & K7.8 & $26.8 \pm 0.8$ & 10.01 & 8 & $3890_{-90}^{+100}$ & $0.15 \pm 0.03$ & $370 \pm 50$ & $2.1 \pm 0.3$ & $1,2,3$ \\
\hline AU Mic & GJ 803 & M0.7 & $9.9 \pm 0.1$ & 8.81 & 9 & $3750_{-70}^{+70}$ & $0.07 \pm 0.01$ & $120 \pm 60$ & $-0.1 \pm 0.7$ & $1,2,3$ \\
\hline GJ 3305 & $\cdots$ & M1.0 & $29.4 \pm 0.3$ & 10.59 & 5 & $3710_{-70}^{+70}$ & $0.13 \pm 0.02$ & $110 \pm 60$ & $-0.4 \pm 0.7$ & 3 \\
\hline $\mathrm{BD}-17^{\circ} 6128 \mathrm{~B}$ & HD 358623 B & M2.9 & $45.7 \pm 1.6^{\mathrm{a}}$ & $\cdots$ & $20^{\mathrm{d}}$ & $3390_{-70}^{+70}$ & $0.05 \pm 0.01$ & $130 \pm 60$ & $-0.2 \pm 0.7$ & 5 \\
\hline HD $155555 \mathrm{C}$ & $\cdots$ & M3.5 & $31.4 \pm 0.5^{\mathrm{e}}$ & 12.71 & 6 & $3340_{-70}^{+70}$ & $0.05 \pm 0.01$ & $<30$ & $<-1.4$ & 2 \\
\hline GJ $799 \mathrm{~N}$ & AT Mic & M4.4 & $10.7 \pm 0.4$ & 11.02 & 10 & $3210_{-70}^{+70}$ & $0.04 \pm 0.01$ & $<10$ & $<-1.4$ & 2,3 \\
\hline GJ $799 \mathrm{~S}$ & AT Mic & M4.4 & $10.7 \pm 0.4$ & 11.02 & 16 & $3210_{-70}^{+70}$ & $0.04 \pm 0.02$ & $<10$ & $<-1.4$ & 2,3 \\
\hline
\end{tabular}

Notes. Except where noted, distances are from the updated Hipparcos catalog (van Leeuwen 2007), $V$ magnitudes are from Zuckerman et al. (2001), and the other data are our own measurements. The given $v \sin i$ is the average of the literature values cited in the last column. (1) $v$ sin $i$ given in Zuckerman et al. (2001), (2) $v \sin i$ given in Torres et al. (2006), (3) $v \sin i$ given in Scholz et al. (2007), (4) Spectral type from Zuckerman et al. (2001), (5) Luminosity from Neuhäuser et al. (2002)

a Assuming that $\mathrm{BD}-17^{\circ} 6128 \mathrm{~A}$ and $\mathrm{B}$ have the same parallax as their companion HD 199143.

b Value for the combined system.

${ }^{c}$ We give the corrected uncertainty in the luminosity of $\mathrm{BD}-17^{\circ} 6128 \mathrm{~A}$ (R. Neuhäuser 2009 , private communication)

d The $v \sin i$ of $\mathrm{BD}-17^{\circ} 6128 \mathrm{~B}$ is not given in the literature. The value given here was estimated from a rotationally broadened stellar template (Section 3 ).

e Assuming that HD $155555 \mathrm{C}$ is at the same distance as HD $155555 \mathrm{~A} / \mathrm{B}$.

of growth given in Soderblom et al. (1993) and Palla et al. (2007) to convert the measured EWs to lithium abundances, following the method used by Sestito et al. (2008). We assumed $\log g=4.5$, which is appropriate for late-type stars descending onto the main sequence. We linearly interpolated the curves of growth in $A(\mathrm{Li})-\mathrm{EW}(\mathrm{Li}$ I) space and used a linear extrapolation for abundances $<0.0$ dex. For the three stars with effective temperature $3600 \mathrm{~K}<T_{\text {eff }}<4000 \mathrm{~K}$, we linearly interpolated between the two tables. For the stars for which we have only an upper limit to the EW, the calculated abundance is necessarily also an upper limit. The quoted uncertainties are the result of individually perturbing both the effective temperatures and EW(Li I)s within their uncertainties and adding the effects on the derived abundances in quadrature. The results are given in Table 1.

Because HD $155555 \mathrm{~A} / \mathrm{B}$ is an $\mathrm{SB}$, we were not able to determine its lithium abundance from the EW(Li I)s. The height of the continuum is roughly twice what would be measured for each star individually; therefore, the lithium EWs of the individual stars would be larger than the EWs given in Table 1, which are measured from the combined spectrum. We use the abundances given in Randich et al. (1993) of 3.5 for HD $155555 \mathrm{~A}$ and 3.7 for HD $155555 \mathrm{~B}$. Based on the discussion in that paper and in Pasquini et al. (1991), we assume an uncertainty of \pm 0.3 dex on those abundances.

\subsection{Lithium Depletion Boundary}

We did not detect the LDB of the BPMG. The latest-type stars in our sample, HD 155555 C, GJ 799 N, and GJ 799 S, showed no observable lithium lines with very sensitive upper limits (Figure 1). The LDB of the BPMG has been detected in two binary systems that are members of this group: HIP 112312 A/B and V343 Nor A/B (Song et al. 2002, 2003). The 6707.8 $\mathrm{Li}$ line was detected in the lower-mass stars in these binaries whose spectral types are M4.5 and M5, respectively. The stars in our sample with non-detections of the lithium line have slightly earlier spectral types, which is consistent with the detected LDB. Comparison of the data with evolutionary models (see below; Figure 3) implies an LDB age of $\gtrsim 30$ Myr. Our data support the conclusion of Song et al. (2002) that there is a discrepancy between the LDB age of this group and the proposed age from $\mathrm{H}-\mathrm{R}$ diagram isochrones of $12_{-4}^{+8}$ Myr (Zuckerman et al. 2001).

\subsection{Comparison to Other Work}

To date, two other groups have published lithium abundances and effective temperatures for some members of the BPMG (Torres et al. 2006; Mentuch et al. 2008). Given the variation in effective temperatures and lithium abundances measured by each group, our data are consistent with previous measurements. Overall, we see the same trend as other groups with lithium decreasing as a function of decreasing spectral type and only upper limits in lithium abundance measured for GJ $799 \mathrm{~N}$ and S.

\subsection{Comments on Individual Objects}

$$
\text { 3.6.1. } C D-64^{\circ} 1208
$$

Examination of the $6707.8 \AA \mathrm{Li}$ I line and the $\mathrm{Ca}$ I line at $6717.7 \AA$ suggests that CD $-64^{\circ} 1208$ is a double-lined SB (Figure 2). Cross-correlation of the spectrum with radial velocity (RV) standards of known velocity and similar spectral type gives velocities of $-28 \pm 8 \mathrm{~km} \mathrm{~s}^{-1}$ for the secondary and $43 \pm 5 \mathrm{~km} \mathrm{~s}^{-1}$ for the primary. Based on the height of the cross-correlation peaks, the secondary has roughly $70 \%-80 \%$ the luminosity of the primary. We note that Torres et al. (2006) also flag this system as "SB?," i.e., a possible spectroscopic binary. Our derived spectral type of K7 agrees with that of Riaz et al. (2006) and is similar to the K5 spectral type found by Torres et al. (2006). We note that the $v \sin i$ of $110 \mathrm{~km} \mathrm{~s}^{-1}$ measured by Torres et al. (2006) is likely an overestimate. The rotation is rapid, but some of the line broadening is due to the velocity separation of the two 

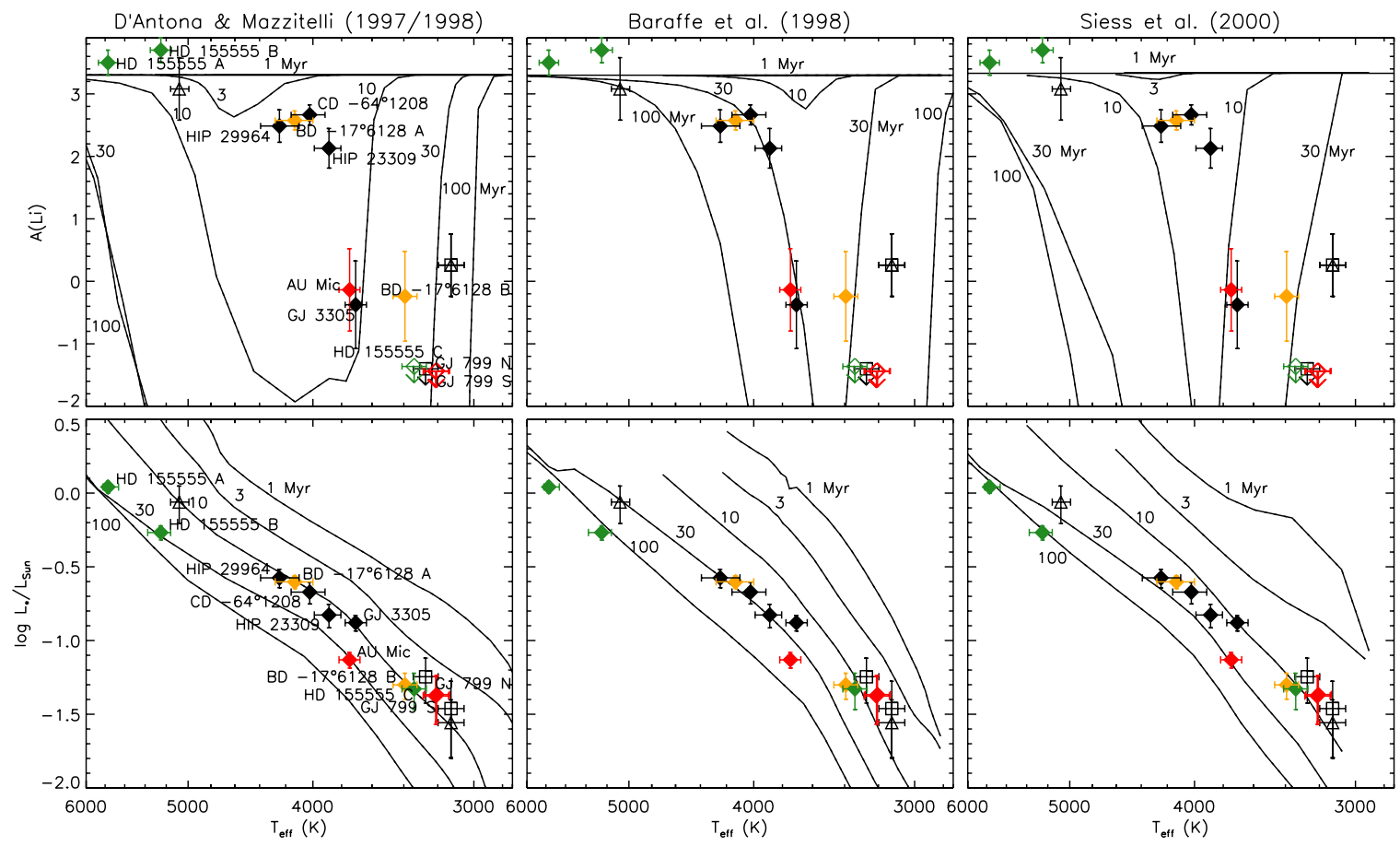

Figure 3. Comparison of the data and theoretical models. The data are overlaid on three sets of models: D'Antona \& Mazzitelli (1997, 1998), Baraffe et al. (1998), and Siess et al. (2000). The results of this work are shown as diamonds. Single stars are shown in black and systems with multiple stars are shown in color (HD 155555 A/B/C, green; BD $-17^{\circ} 6128 \mathrm{~A} / \mathrm{B}$, orange; and AU Mic/GJ $799 \mathrm{~N} / \mathrm{S}$, red). Upper limits are shown as open diamonds with arrows. The squares show HD 112312 A/B (Song et al. 2002) and the triangles show V343 Nor A/B (Torres et al. 2006). Note that the luminosities and effective temperatures for GJ 799 N/S are similar, so the two stars appear to be a single data point. Likewise, the lithium abundances and temperatures for HD 112312 B and V343 Nor B are the same. Note that the ages predicted by the location on the H-R diagram and by the location on the corresponding lithium abundance plot differ for any individual star. Taking the stars as a group, it is apparent that the models do not correctly predict the observed patterns of lithium depletion. This is also true of the stars in individual multiple systems whose components must be coeval.

stars. If the luminosities and effective temperatures of the two components are similar, as seems likely from the spectrum, the EWs of the individual lithium line and the lithium abundances of the individual components should be roughly the same as those given in Table 1 .

\subsection{2. $A U M i c$}

If $\mathrm{AU}$ Mic is the same age as the other members of the BPMG, it appears to be underluminous in the $\mathrm{H}-\mathrm{R}$ diagram (Figure 3 ). We observe $\log \left(L / L_{\odot}\right)=-1.15$, but from the figure we might expect a value closer to $\log \left(L / L_{\odot}\right)=-0.95$ for it to lie on the same $\mathrm{H}-\mathrm{R}$ diagram isochrone as the other BPMG members. AU Mic is known to have an edge-on debris disk (cf. Kalas et al. 2004), which could lead to some extinction. Given the difference between our observed and expected values for the luminosity, if $\mathrm{AU}$ Mic is coeval on the $\mathrm{H}-\mathrm{R}$ diagram, then we estimate an extinction due to the debris disk of $A_{V} \sim 0.5 \mathrm{mag}$. Since this extinction is not well characterized, and it is dependent on an assumption of coevality, we do not take it into account in our analysis. This proposed extinction should not affect our measurement of the effective temperature since that is determined from the spectral type which is measured over a small range in wavelength. However, it does affect any age determined from the $\mathrm{H}-\mathrm{R}$ diagram position. If the luminosity we observe is less than the intrinsic luminosity of AU Mic, we would overestimate the age of the star (see Figure 3).

\section{COMPARISON TO EVOLUTIONARY MODELS}

In Figure 3, we plot our data against three sets of evolutionary models: D'Antona \& Mazzitelli (1997, 1998), Baraffe et al.
(1998), and Siess et al. (2000). The Siess et al. (2000) models include the effects of convective overshooting. Known multiple systems are plotted in color. We also plot HIP $112312 \mathrm{~A} / \mathrm{B}$ and V343 Nor A/B, two systems that cross the LDB. We calculated luminosities and effective temperatures for these stars in the same manner as for our sample, using published spectral types and $V$ magnitudes (Zuckerman et al. 2001; Song et al. 2003; Torres et al. 2006). We used the lithium abundances from Torres et al. (2006). Neither Song et al. (2002) nor Torres et al. (2006) quote a lithium abundance for HIP $112312 \mathrm{~A}$. Since the spectral type and the upper limit in the EW of this star given in Song et al. (2002) are similar to those of GJ 799 N, we assign HIP 112312 A an upper limit of $A(\mathrm{Li})=-1.4$ dex.

To explore the differences between the models and our data, we independently determine ages for each star in two ways: from its position on the $\mathrm{H}-\mathrm{R}$ diagram and from its position relative to the lithium depletion isochrones. We estimate the uncertainty of the $\mathrm{H}-\mathrm{R}$ diagram age by perturbing the effective temperatures and luminosities within their uncertainties and adding the resulting age errors in quadrature. Because the measured lithium abundance is correlated with effective temperature, we estimate the uncertainties in the lithium depletion age by simultaneously varying the effective temperatures and lithium abundances within their uncertainties and taking the extreme values for the age as a measure of its uncertainty. These data are given in Table 2 and shown in Figure 4. This figure shows that the ages calculated from the H-R diagram are roughly consistent with a single age, but the ages measured from the lithium depletion have systematic trends. The trend of increasing age with spectral type found using the D'Antona \& Mazzitelli $(1997,1998)$ and Siess et al. (2000) lithium depletion isochrones simply reflects 
HRD

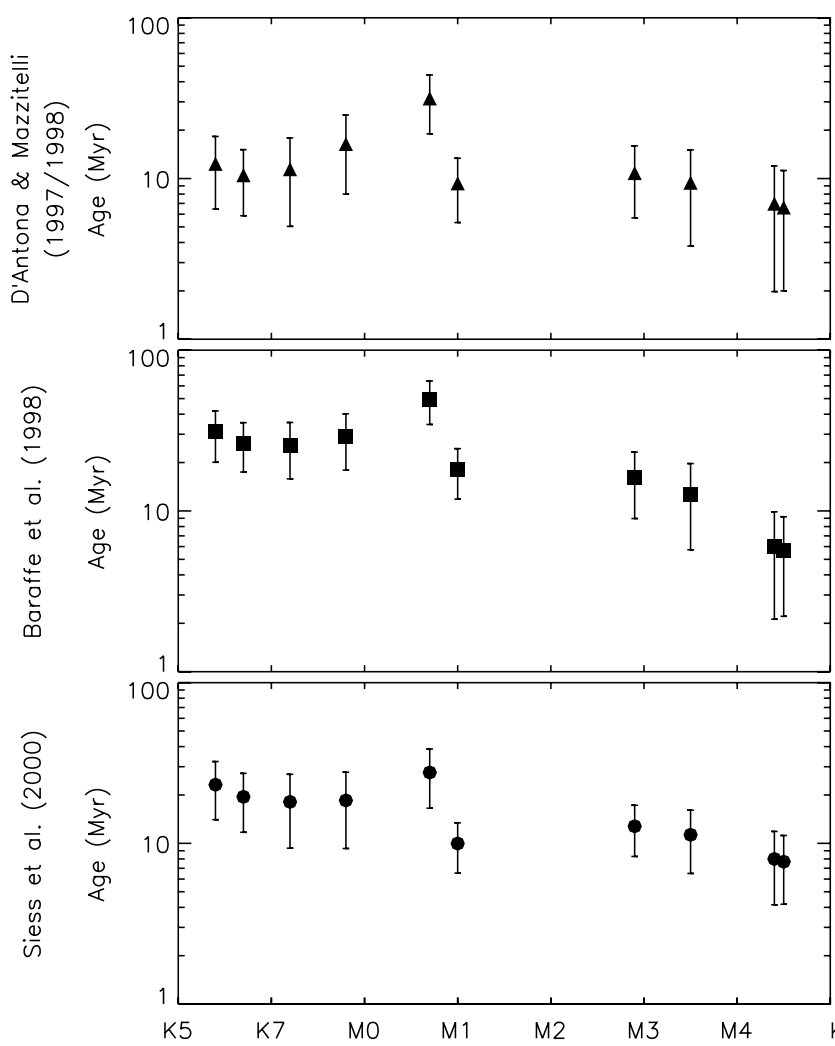

$A(L i)$

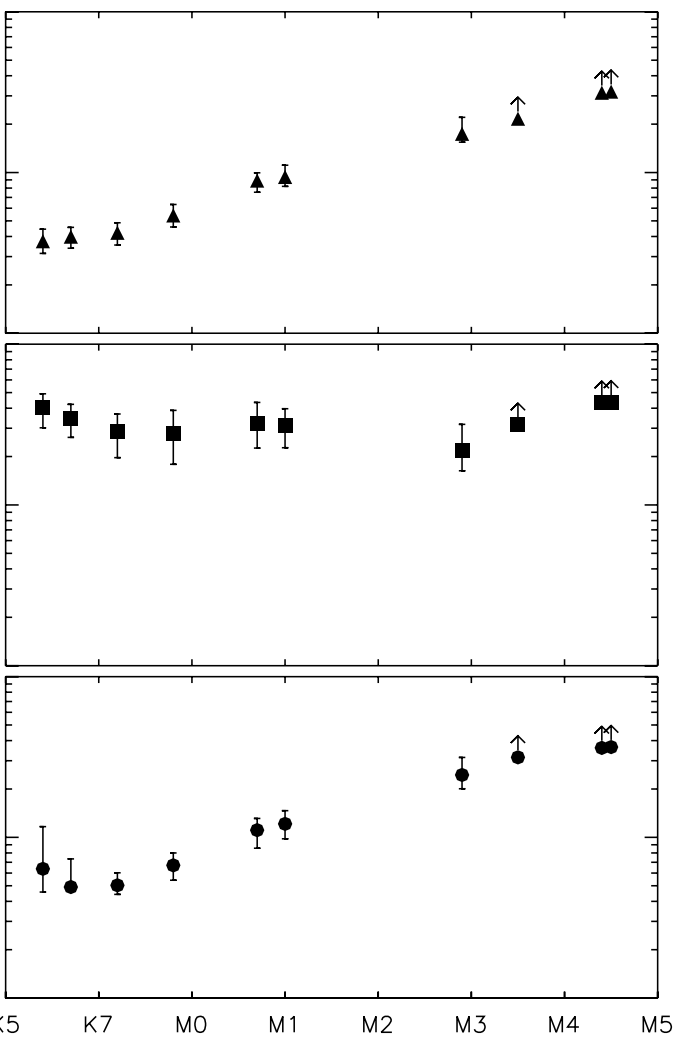

Figure 4. Age as a function of spectral type, calculated for individual stars from different models. The left column shows ages calculated from the $\mathrm{H}-\mathrm{R}$ diagram and the right column shows ages calculated from the lithium depletion isochrones. Since GJ $799 \mathrm{~N}$ and S have the same spectral type, the points for GJ $799 \mathrm{~S}$ have been displaced by +0.1 in spectral type for clarity.

Table 2

Ages in Myr Calculated from Different Models and Isochrones

\begin{tabular}{|c|c|c|c|c|c|c|}
\hline \multirow[t]{2}{*}{ Star } & \multicolumn{2}{|c|}{$\begin{array}{c}\text { D'Antona \& Mazzitelli } \\
(1997,1998)\end{array}$} & \multicolumn{2}{|c|}{ Baraffe et al. (1998) } & \multicolumn{2}{|c|}{ Siess et al. (2000) } \\
\hline & HRD & $\mathrm{A}(\mathrm{Li})$ & HRD & $\mathrm{A}(\mathrm{Li})$ & $\mathrm{HRD}$ & $\mathrm{A}(\mathrm{Li})$ \\
\hline HIP 29964 & $12_{-6}^{+6}$ & $4_{-1}^{+1}$ & $31_{-11}^{+11}$ & $40_{-13}^{+10}$ & $23_{-9}^{+9}$ & $6_{-2}^{+6}$ \\
\hline $\mathrm{BD}-17^{\circ} 6128 \mathrm{~A}$ & $10_{-5}^{+5}$ & $4_{-1}^{+1}$ & $26_{-9}^{+9}$ & $35_{-9}^{+9}$ & $19_{-8}^{+8}$ & $5_{-0}^{+3}$ \\
\hline HIP 23309 & $16_{-8}^{+8}$ & $5_{-1}^{+1}$ & $29_{-11}^{+11}$ & $28_{-11}^{+12}$ & $18_{-9}^{+9}$ & $7_{-1}^{+1}$ \\
\hline $\mathrm{CD}-64^{\circ} 1208$ & $11_{-6}^{+6}$ & $4_{-1}^{+1}$ & $26_{-10}^{+10}$ & $29_{-11}^{+10}$ & $18_{-9}^{+9}$ & $5_{-1}^{+1}$ \\
\hline AU Mic & $32_{-13}^{+13}$ & $9_{-2}^{+1}$ & $49_{-15}^{+15}$ & $32_{-11}^{+12}$ & $28_{-11}^{+11}$ & $11_{-3}^{+2}$ \\
\hline GJ 3305 & $9_{-4}^{+4}$ & $9_{-1}^{+2}$ & $18_{-6}^{+6}$ & $31_{-9}^{+10}$ & $10_{-3}^{+3}$ & $12_{-2}^{+3}$ \\
\hline $\mathrm{BD}-17^{\circ} 6128 \mathrm{~B}$ & $11_{-5}^{+5}$ & $17_{-2}^{+5}$ & $16_{-7}^{+7}$ & $22_{-6}^{+10}$ & $13_{-4}^{+4}$ & $24_{-4}^{+7}$ \\
\hline HD $155555 \mathrm{C}$ & $9_{-6}^{+6}$ & $>22$ & $13_{-7}^{+7}$ & $>32$ & $11_{-5}^{+5}$ & $>31$ \\
\hline GJ $799 \mathrm{~N}$ & $7_{-5}^{+5}$ & $>31$ & $6_{-4}^{+4}$ & $>43$ & $8_{-4}^{+4}$ & $>36$ \\
\hline GJ $799 \mathrm{~S}$ & $7_{-5}^{+5}$ & $>32$ & $6_{-4}^{+4}$ & $>44$ & $8_{-4}^{+4}$ & $>36$ \\
\hline
\end{tabular}

the fact that the data cross the isochrones instead of lying parallel to them, as seen in Figure 3. Although the Baraffe et al. (1998) models are the closest to predicting a coeval group using lithium depletion, they still fail to accurately represent the latest-type stars in the sample. Even though the predicted ages are similar, the pattern of lithium depletion seen in the Baraffe et al. (1998) models is the opposite of what is seen in our data. In particular, these lithium depletion isochrones indicate that the LDB occurs between $3400 \mathrm{~K}$ and $3200 \mathrm{~K}$ for the $30 \mathrm{Myr}$ isochrone, which approximates our data. Thus, we expect that stars at the cooler end of the temperature range 3200-3400 K should have a higher lithium abundance than stars at the warmer end. In contrast, we observe that HD $155555 \mathrm{C}$, GJ $799 \mathrm{~N}$, and GJ $799 \mathrm{~S}$ with $T_{\text {eff }} \sim$ $3300 \mathrm{~K}, 3200 \mathrm{~K}$, and $3200 \mathrm{~K}$, respectively, have only upper limits in lithium abundance, whereas the warmer star BD $-17^{\circ} 6128 \mathrm{~B}$ $\left(T_{\text {eff }} \sim 3400 \mathrm{~K}\right)$ has a larger lithium abundance.

From these two methods for measuring ages, we observe two systematic trends in the data. First, the ages of the mid$\mathrm{M}$ dwarfs calculated from the $\mathrm{H}-\mathrm{R}$ diagram isochrones are slightly younger than the ages of the warmer stars. This effect has also been noted by Stassun et al. (2004) in the ages of binary components and has been recognized more generally as a problem for PMS evolutionary models by Hillenbrand et al. (2008). The second trend is that the ages of the mid-M stars 


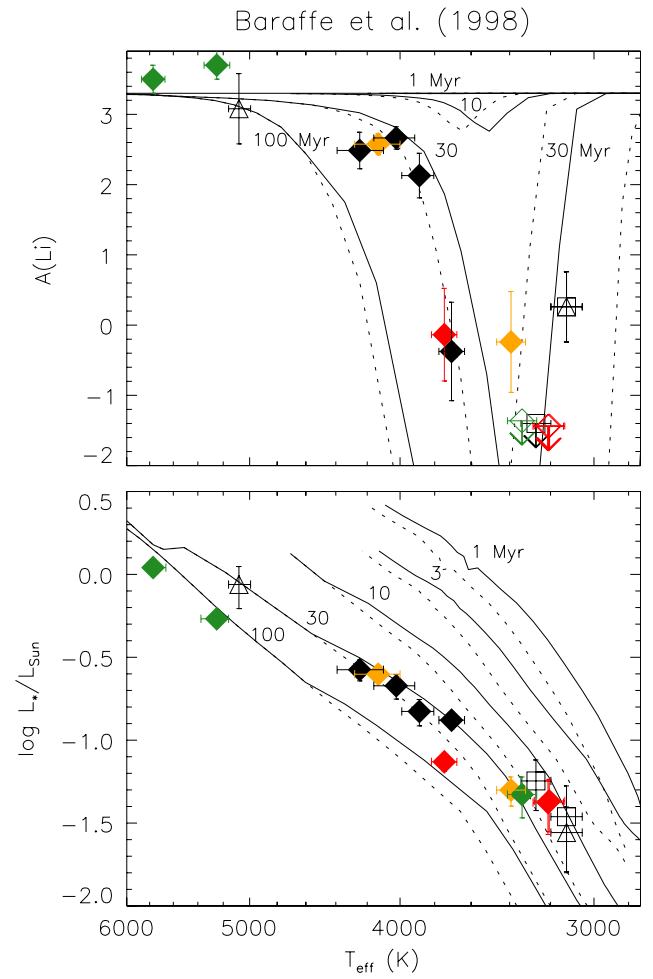

Figure 5. Baraffe et al. (1998) evolutionary models with artificially inflated radii. The solid lines show the isochrones with an ad hoc correction assuming that for $T_{\text {eff }} \lesssim 4200 \mathrm{~K}$ the radii should be increased by $10 \%$. The dotted lines show the original model isochrones from Figure 3. The other symbols are the same as those shown in Figure 3.

(A color version of this figure is available in the online journal.)

calculated from their lithium depletion are older than the ages of the warmer stars.

While the H-R diagram isochrones match our data well and the stars are roughly coeval, none of the evolutionary models have lithium depletion isochrones consistent with a coeval group. If we consider each multiple star system independently of the group as a whole, we find similar inconsistencies in the predicted ages for the hotter and cooler stars in each binary. Since the two stars in each binary are almost certain to be coeval within $<1 \mathrm{Myr}^{6}{ }^{6}$ this indicates that the mismatch between our data and the isochrones is not the result of contamination by unrelated stars or an extended period of star formation. In general, the models seem to have difficulty describing the lithium depletion of the lowest-mass stars. In particular, the modeled rise in lithium abundance at lower temperatures (the LDB) occurs at temperatures too high for our stars in all of the evolutionary models. Given the nominal H-R diagram age, this means that an estimate of the age of the group from the LDB will be systematically older than an age from fitting the $\mathrm{H}-\mathrm{R}$ diagram regardless of the model used.

\section{LARGER M-DWARF RADII TO THE RESCUE?}

We have shown that the pattern of Li depletion seen in the BPMG stars is at odds with the predictions of PMS Li depletion

\footnotetext{
6 The multiplicity frequency of stars at the youngest observable PMS ages is comparable to or greater than that among field stars (Duchêne et al. 2007, and references therein), indicating that binary formation is part of the star

formation process. Thus, we can consider binary systems to be $N=2$, coeval clusters to within $<1 \mathrm{Myr}$, although there are hints of smaller age differences (Stassun et al. 2008).
}

models, in the sense that the models underpredict the amount of Li depletion in the M stars in our sample. The evolutionary models have a certain amount of intrinsic uncertainty due to assumptions about mixing length, equation of state, opacity, and atmosphere and boundary conditions, whose effects on lithium depletion are discussed in some detail in Burke et al. (2004). Without a specific physical reason to change the assumptions, it is difficult to decide which parameter or combination of parameters should be changed in order to fit the lithium data. However, there is an increasingly large body of observations (see below) that evolutionary models tend to underpredict the radii of low-mass stars, particularly $\mathrm{M}$ stars, which may point the way toward the types of modifications to the models necessary to bring the $\mathrm{H}-\mathrm{R}$ diagram ages and $\mathrm{Li}$ depletion ages into agreement.

Changes in stellar radii could affect the observed pattern of Li depletion in two ways: by actually changing Li depletion as a function of mass, and/or by altering the effective temperatures of stars, thus shifting them relative to the model isochrones. Regarding actual changes in Li depletion, the work of King et al. (2010) suggests a connection between Li depletion and stellar radii. They propose that a range in radii at a given stellar mass (perhaps due to a range in rotation rates and/or chromospheric activity) would lead to a range of interior temperature profiles and could explain the dispersion in lithium depletion in the Pleiades.

However, even if the models are essentially correct in terms of the interior conditions for a given mass, which largely govern the rate of Li depletion, there could still be a disagreement between the model predictions and the observations if the models do not correctly predict the surface conditions, e.g., the radius or effective temperature. We suggest here that current data show evidence of exactly this sort of disagreement.

There is mounting observational evidence that main-sequence stellar evolutionary models predict radii for $\mathrm{M}$ dwarfs that are $10 \%-20 \%$ smaller than the observed radii. This effect is seen in stellar angular diameters measured interferometrically (Lane et al. 2001; Ségransan et al. 2003; Berger et al. 2006), in radii determined from eclipsing binaries (López-Morales 2007; Ribas et al. 2008; Fernandez et al. 2009), and in radii determined from detailed modeling of multi-band photometry to measure $L_{\mathrm{Bol}}$ and $T_{\text {eff }}$ (Mullan \& MacDonald 2001; Casagrande et al. 2008). There is still some question as to whether this discrepancy exists only for stars that are the most magnetically active (Demory et al. 2009), or if it applies to all M dwarfs (Casagrande et al. 2008). There does seem to be at least some differential effect from stellar activity (Mullan \& MacDonald 2001; Morales et al. 2008), and models incorporating the effects of stellar activity are better able to reproduce the observed radii (Chabrier et al. 2007). For the present work, the question is moot as the stars in our sample (and indeed all late-type PMS stars) are very active, with large X-ray luminosities and active chromospheres.

The discrepancy between the true radii and the radii predicted by models very likely exists in the PMS phase as well, if it is due to effects of magnetic activity and/or missing opacity sources in the models. If this discrepancy is present, how would it manifest itself? Here, we argue that the effect would be similar to what we observe, and correcting for this would bring $\mathrm{H}-\mathrm{R}$ diagram ages and $\mathrm{Li}$ depletion ages closer together. Corrected model isochrones for a given age would need to move to lower $T_{\text {eff }}$, increasing the age inferred from the H-R diagram and decreasing the age inferred from $\mathrm{Li}$ depletion for a given star. 
First consider the H-R diagram. PMS stars change position in the H-R diagram as they age precisely because their radii are changing, as the stars descend (contract) toward the main sequence. If, at any given age, the true radii of stars are larger, then the model isochrones for that age need to move up and to the right. Thus, the $\mathrm{H}-\mathrm{R}$ diagram age of a given star (of fixed, observed $T_{\text {eff }}$ and $\left.L_{\mathrm{Bol}}\right)$ inferred from these corrected models would be older than before. Put another way, if radii are inflated by effects other than youth, it will take longer for a star to contract to a given radius, and thus to reach a given position on the $\mathrm{H}-\mathrm{R}$ diagram.

Now, consider the $A(\mathrm{Li})-T_{\text {eff }}$ plane. The amount of $\mathrm{Li}$ depletion at a given age is primarily driven by a star's mass, which sets its interior conditions. Inflating the radius of a star will cause that star to have a cooler surface, i.e., a lower $T_{\text {eff }}$. Thus, corrected model isochrones would need to shift to the right for a given $A(\mathrm{Li})$ and age. Put another way, a star with an inflated radius will have a hotter interior than we might otherwise expect given its surface appearance, and thus will exhibit more $\mathrm{Li}$ depletion at a given age and $T_{\mathrm{eff}}$ than would be predicted if the radius were not modified. This matches well with the enhanced Li depletion we observe for the cooler stars.

Calculating new PMS evolutionary models incorporating these effects is beyond the scope of this work, but we can explore these effects to a limited degree by introducing an ad hoc shift to the existing model isochrones. As a test of this idea, we shifted the model isochrones of Baraffe et al. (1998) and compared them to our observations of $A(\mathrm{Li})$ and $T_{\text {eff }}$ for the BPMG stars. Based on the observations of M stars discussed above, we assumed that the larger radii are present only for stars cooler than $\sim 4200 \mathrm{~K}$ (Casagrande et al. 2008), and that the model radii should be inflated by $10 \%$. As shown in Figure 5, these corrections to the models do indeed bring the inferred $\mathrm{Li}$ depletion and $\mathrm{H}-\mathrm{R}$ diagram ages of the cooler stars into better agreement, though some discrepancies remain. Clearly, a more systematic treatment of this effect is needed, perhaps taking into account effects of different activity levels as well as different masses.

To close, we note that one implication of this result is that neither the LDB ages nor the $\mathrm{H}-\mathrm{R}$ diagram ages of $\mathrm{M}$ stars are correct, but that the answer lies somewhere between the two.

\section{CONCLUSIONS}

We have measured lithium abundances and effective temperatures for ten members of the BPMG. We compare these abundances to the predictions of the evolutionary models of D'Antona \& Mazzitelli (1997, 1998), Baraffe et al. (1998), and Siess et al. (2000). We find that while the H-R diagram isochrones of these models reproduce our data fairly well, the lithium depletion isochrones do not. In particular, the models predict less lithium depletion for the latest-type stars than we observe. Thus, the ages determined based on the lithium abundances are older than the ages determined from the $\mathrm{H}-\mathrm{R}$ diagram.

The most striking result is the non-detection of lithium in the latest-type members (M3.5-M5) of this group. The lack of an observable lithium line in HD 155555 C, GJ 799 N, and GJ 799 S indicates that lithium depletion proceeds at a much faster rate than is predicted in the theoretical models given their spectral types. One potential clue lies in the discrepancy between the observed radii of $\mathrm{M}$ dwarfs and the radii predicted by models.

Regardless of the physical mechanism, it appears that mid-M stars deplete their lithium rapidly. The presence of the lithium line at $6707.8 \AA$ in a star of this type remains a clear indicator of youth and suggests an age younger than that of the BPMG, while the lack of a detectable line is not a strong indicator that a star is old.

We thank the referee for useful comments that improved this work. We thank Marc Pinsonneault, Keivan Stassun, and Peter Hauschildt for helpful conversations. We also thank Christine Johnas and Blair Reaser for early input into this work. E.J. gratefully acknowledges the support of a Eugene M. Lang Faculty Fellowship from Swarthmore College, and NSF grant AST-0307830. J.C.Y. acknowledges the support of NSF grant AST-0757888.

\section{REFERENCES}

Baraffe, I., Chabrier, G., Allard, F., \& Hauschildt, P. H. 1998, A\&A, 337, 403 Barrado y Navascués, D., Stauffer, J. R., \& Jayawardhana, R. 2004, ApJ, 614, 386

Basri, G., Marcy, G. W., \& Graham, J. R. 1996, ApJ, 458, 600

Berger, D. H., et al. 2006, ApJ, 644, 475

Bildsten, L., Brown, E. F., Matzner, C. D., \& Ushomirsky, G. 1997, ApJ, 482, 442

Burke, C. J., Pinsonneault, M. H., \& Sills, A. 2004, ApJ, 604, 272

Casagrande, L., Flynn, C., \& Bessell, M. 2008, MNRAS, 389, 585

Chabrier, G., Gallardo, J., \& Baraffe, I. 2007, A\&A, 472, L17

D’Antona, F., \& Mazzitelli, I. 1997, Mem. Soc. Astron. Ital., 68, 807

D’Antona, F., \& Mazzitelli, I. 1998, in ASP Conf. Ser. 134, Brown Dwarfs and Extrasolar Planets, ed. R. Rebolo, E. L. Martin, \& M. R. Zapatero Osorio (San Francisco, CA: ASP), 442

Demory, B., et al. 2009, A\&A, 505, 205

Duchêne, G., Delgado-Donate, E., Haisch, K. E. Jr., Loinard, L., \& Rodríguez, L. F. 2007, in Protostars and Planets V, ed. B. Reipurth, D. Jewitt, \& K. Keil (Tucson, AZ: Univ. Arizona Press), 379

Fernandez, J. M., et al. 2009, ApJ, 701, 764

Hillenbrand, L. A., Bauermeister, A., \& White, R. J. 2008, in ASP Conf. Ser. 384, 14th Cambridge Workshop on Cool Stars, Stellar Systems, and the Sun, ed. G. van Belle (San Francisco, CA: ASP), 200

Jeffries, R. D. 2000, in ASP Conf. Ser. 198, Stellar Clusters and Associations: Convection, Rotation, and Dynamos, ed. R. Pallavicini, G. Micela, \& S. Sciortino (San Francisco, CA: ASP), 245

Jeffries, R. D., \& Naylor, T. 2001, in ASP Conf. Ser. 243, From Darkness to Light: Origin and Evolution of Young Stellar Clusters, ed. T. Montmerle \& P. André (San Francisco, CA: ASP), 633

Jeffries, R. D., \& Oliveira, J. M. 2005, MNRAS, 358, 13

Kalas, P., Liu, M. C., \& Matthews, B. C. 2004, Science, 303, 1990

Kenyon, S. J., \& Hartmann, L. 1995, ApJS, 101, 117

King, J. R., Schuler, S. C., Hobbs, L. M., \& Pinsonneault, M. H. 2010, ApJ, 710,1610

Kirkpatrick, J. D., Henry, T. J., \& McCarthy, D. W., Jr. 1991, ApJS, 77, 417

Lane, B. F., Boden, A. F., \& Kulkarni, S. R. 2001, ApJ, 551, L81

López-Morales, M. 2007, ApJ, 660, 732

Luhman, K. L. 1999, ApJ, 525, 466

Manzi, S., Randich, S., de Wit, W. J., \& Palla, F. 2008, A\&A, 479, 141

Mentuch, E., Brandeker, A., van Kerkwijk, M. H., Jayawardhana, R., \& Hauschildt, P. H. 2008, ApJ, 689, 1127

Morales, J. C., Ribas, I., \& Jordi, C. 2008, A\&A, 478, 507

Mullan, D. J., \& MacDonald, J. 2001, ApJ, 559, 353

Navascués, D., Stauffer, J. R., \& Patten, B. M. 1999, ApJ, 522, L53

Neuhäuser, R., Guenther, E., Mugrauer, M., Ott, T., \& Eckart, A. 2002, A\&A, 395, 877

Palla, F., Randich, S., Pavlenko, Y. V., Flaccomio, E., \& Pallavicini, R. 2007, ApJ, 659, L41

Pasquini, L., Cutispoto, G., Gratton, R., \& Mayor, M. 1991, A\&A, 248, 72

Randich, S., Gratton, R., \& Pallavicini, R. 1993, A\&A, 273, 194

Reid, I. N., Hawley, S. L., \& Gizis, J. E. 1995, AJ, 110, 1838

Riaz, B., Gizis, J. E., \& Harvin, J. 2006, AJ, 132, 866

Ribas, I., Morales, J. C., Jordi, C., Baraffe, I., Chabrier, G., \& Gallardo, J. 2008 , Mem. Soc. Astron. Ital., 79, 562

Scholz, A., Coffey, J., Brandeker, A., \& Jayawardhana, R. 2007, ApJ, 662, 1254 Ségransan, D., Kervella, P., Forveille, T., \& Queloz, D. 2003, A\&A, 397, L5 Sestito, P., Palla, F., \& Randich, S. 2008, A\&A, 487, 965

Siess, L., Dufour, E., \& Forestini, M. 2000, A\&A, 358, 593 
Soderblom, D. R., Jones, B. F., Balachandran, S., Stauffer, J. R., Duncan, D. K., Fedele, S. B., \& Hudon, J. D. 1993, AJ, 106, 1059

Song, I., Bessell, M. S., \& Zuckerman, B. 2002, ApJ, 581, L43

Song, I., Zuckerman, B., \& Bessell, M. S. 2003, ApJ, 599, 342

Stassun, K. G., Mathieu, R. D., Cargile, P. A., Aarnio, A. N., Stempels, E., \& Geller, A. 2008, Nature, 453, 1079

Stassun, K. G., Mathieu, R. D., Vaz, L. P. R., Stroud, N., \& Vrba, F. J. 2004, ApJS, 151,357
Stauffer, J. R., et al. 1999, ApJ, 527, 219

Torres, C. A. O., Quast, G. R., da Silva, L., de La Reza, R., Melo, C. H. F., \& Sterzik, M. 2006, A\&A, 460, 695

van Leeuwen, F. (ed.) 2007, in Astrophysics and Space Science Library 350, Hipparcos, the New Reduction of the Raw Data (Dordrecht: Springer)

White, R. J., \& Hillenbrand, L. A. 2005, ApJ, 621, L65

Zuckerman, B., \& Song, I. 2004, ARA\&A, 42, 685

Zuckerman, B., Song, I., Bessell, M. S., \& Webb, R. A. 2001, ApJ, 562, L87 